\title{
Un evento catastrofico: una sfida per l'attivazione di strutture di conoscenza e di azione
}

\author{
Roberta Bisi ${ }^{\bullet}$
}

\section{Riassunto}

L'articolo analizza, partendo dalle relazioni di consulenza tecnica di ufficio medico-legale e psichiatrica, il percorso biografico e la rappresentazione della condannata AMF.

Un'attenzione particolare viene rivolta ad alcuni degli strumenti psicodiagnostici utilizzati per delineare il profilo di personalità di AMF e, di conseguenza, l'impegno volto a valorizzare ed affermare la dignità dei sentimenti e delle relazioni affettive, utili per ridare senso alla propria appartenenza sociale, nel tentativo di conciliare le ragioni del cuore e le passioni della ragione.

\section{Résumé}

À partir du rapport d'expertise médico-légale et psychiatrique, l'article analyse le récit de vie d'AMF et la représentation de soi.

Une attention particulière est portée à certaines méthodes psychodiagnostiques utilisées pour établir le profil de la personnalité d'AMF dans le but de mettre en valeur et d'affirmer la dignité des sentiments et des relations affectives. Ces dernières s'avèrent utiles pour redonner sens à son appartenance sociale en cherchant à concilier les raisons du cœur et les passions de la raison.

\section{Abstract}

On the basis of the technical consulting reports concerning the psychiatric clinical forensic examination, the article analyses AMF's biographic background and her self-representation.

A special attention is paid to some of the psychodiagnostic tools used to outline the personality profile of AMF and as a consequence it is taken into consideration the responsibility to appraise and state the dignity of the emotional feelings and the family bonds necessary to give meaning to the individual social identity in the attempt to conciliate the reasons of the heart and the passions of the reason.

Key words: Cogne case; life narrative method; self-representation; psychodiagnostic tools; personality profile.

\section{Collassato in una pozza di sangue.}

"La dottoressa A.S., appena giunta verso le ore 8.32' circa, prestava i primi soccorsi al bambino che appariva "collassato in una pozza di sangue", coperto fino all'inguine o fino alla cintola, comunque, visibile dallo sterno in su, con una ferita importante a livello dell'osso frontale sulla parte destra, da cui usciva materia cerebrale, con altre piccole lesioni sulla parte alta del viso; avvicinato il bambino a sé sul letto, constatava che vi era anche una lesione sulla sinistra dall'occhio, a salire sulla fronte; per rendere meno penosa alla madre la vista del bambino, provvedeva a lavargli il volto - completamente imbrattato di sangue, come il cranio - con acqua prelevata da una bacinella, che appoggiava sul letto, poi gli praticava una iniezione di cortisone. Il bambino gemeva sommessamente, non era dotato di alcun movimento spontaneo, aveva gli occhi socchiusi, le mascelle serrate, la lingua in sede, la bocca sporca di sangue all'interno, ed un colore cutaneo che esprimeva grave stato di shock; aveva polso carotideo, i denti insanguinati, ma non vi era fuoriuscita di sangue dalla bocca ed

\footnotetext{
- Professore ordinario di "Sociologia giuridica della devianza e mutamento sociale", Dipartimento di Sociologia e Diritto dell'Economia, Università di Bologna.
} 
il suo pallore era andato accentuandosi. Vi erano frammenti di materia cerebrale anche nella pozza di sangue che circondava "quasi ad aureola" la testa del bambino, adagiata sul cuscino fortemente imbrattato di sangue e da lei stessa capovolto, affinché la testa non appoggiasse su detto materiale organico (in seguito, aveva precisato che il bimbo era supino, con le braccia distese lungo il corpo, la testa in parte sul bordo inferiore del cuscino ed in parte sul materasso che, anzi, la porzione maggiore della testa - a suo ricordo - era proprio sul materasso ed era una vera e propria maschera di sangue, ...). Aveva, poi, avvicinato il bambino al bordo del letto per poterlo avere più vicino, richiamando il 118 per ribadire la gravità del caso. All'inizio, aveva creduto essersi trattato di incidente, tanto che aveva chiesto a AMF se il bambino era caduto, poi aveva ipotizzato un'implosione per forte pressione endocranica, correlata a non completa saldatura del cranio, possibile in bambini di quella età [.....].

Il bambino, caricato sull'elicottero alle ore 9.19', arrivava al Pronto Soccorso dell'ospedale di Aosta alle ore 9.47', in codice GCS3, con paramenti vitali fortemente alterati e pressoché assenti, intubato ed in ventilazione assistita, con parametri circolatori non valutabili e pupille dilatate: alle ore 9.55, attesa la inefficacia delle manovre rianimatorie, il dr. B. ne dichiarava il decesso, come da referto in atti”.

Questo è il racconto dei fatti riguardanti il ritrovamento, i soccorsi e la dichiarazione di morte di S.L. presenti nella sentenza della prima Corte di Assise di Torino - 2007.

I colloqui effettuati e l'esame delle attività di consulenza tecnica consentono alcune riflessioni circa la presentazione che AMF fa di se stessa. La presentazione di sé, infatti, non è un atto banale, ma è intrisa di tensione poiché mette in gioco l'identità sociale e personale del soggetto. In effetti dai numerosi studi effettuati, e da quelli di Goffman in particolare, sui temi della personalità, dell'interazione sociale e della società sappiamo che quando una persona si presenta agli altri proietta, in parte consapevolmente ed in parte involontariamente, una definizione della situazione in cui l'idea che la persona si fa della situazione medesima costituisce un elemento importante $^{1}$. Presentandosi agli altri, la persona mobilita, in modo più o meno consapevole, una definizione di se stessa costituita da immagine e da rappresentazione di sé. In tal senso, è possibile rifarsi alla concezione del sé operante, working self, inteso come un sottoinsieme di rappresentazioni che sono attivate in relazione alle circostanze sociali e agli stati motivazionali del soggetto. E' a questa peculiare forma di conoscenza di sé che il soggetto si rifà per leggere ed interpretare le sollecitazioni e le informazioni correlate ad un particolare contesto e per programmare le strategie di azione ${ }^{2}$. Ma l'altro non si limita a recepire in modo passivo questa presentazione. Vi reagisce in funzione della sua posizione sociale e della rappresentazione che egli si fa dell'immagine di sé che ha colui che gli sta di fronte.

Questa immagine se la costruisce riferendosi alle differenti appartenenze categoriali, istituzionali ed educative che precedono l'incontro faccia a faccia. E' ciò che è stato definito "ethos prediscorsivo",

\footnotetext{
${ }^{1}$ Goffman E., La mise en scène de la vie quotidienne. La présentation de soi, Les Éditions de minuit, Paris, 1973.

${ }^{2}$ Arcuri L., "Rappresentazione di sé ed elaborazione dell'informazione sociale", in Caprara G.V. (a cura di),
} 
un ethos preliminare che precede appunto la costruzione dell'immagine nel discorso ${ }^{3}$.

Queste brevi osservazioni appaiono necessarie per introdurre alcune note riguardanti la biografia di AMF e la costruzione dell'immagine di sé così come emergono dalla documentazione studiata, divenuta parte integrante della costruzioneprogetto delle consulenze tecniche d'ufficio disposte dal Tribunale di Sorveglianza di Bologna.

\section{Percorsi biografici e rappresentazione di sé.}

AMF si è sempre presentata ai diversi incontri corretta nel contegno, ordinata e curata nell'abbigliamento, aderendo sempre di buon grado ai temi che venivano affrontati nei diversi colloqui. Esponendo le caratteristiche della propria famiglia, AMF mise in rilievo il rapporto con la propria madre in questi termini: "Io ho sempre respirato da mia madre un senso materno molto forte, che è quello che ho anch'io, perché sento come priorità, come donna, la famiglia". AMF dichiara di non aver mai pensato a se stessa e alla carriera perché: "non mi interessa proprio niente, quello che mi gratifica di più e che mi fa stare bene è il tenere la casa, stare con i miei figli, stare con mio marito, fargli trovare quel calore materno, di moglie, familiare che ho respirato da piccola e che dentro di me è infinito, è un qualcosa che mi fa stare bene, è un qualcosa che penso sia lo scopo della mia vita. Quello che ho respirato in casa mia è questo, la normalità dei figli, poi uno fa la scelta di uno, di tanti, però

Personalità e rappresentazione sociale, La Nuova Italia Scientifica, Roma, 1988, pp. 17-32.

3 Haddad G., «Ethos préalable et ethos discursif: l'exemple de Romain Rolland », in R Amossy (sous la direction de), Images de soi dans le discours - La comunque quel senso familiare, quella dedizione di fare proprio la vita che ti è donata e far respirare la bellezza di tutto ciò che è libero".

A questo proposito occorre evidenziare che i fattori, diretti ed indiretti, in grado di influenzare il genitore nei suoi compiti di parenting si ripercuoteranno pure sul rapporto precoce adulto bambino e sull'evoluzione di quest'ultimo. Nella descrizione di Belsky sono tre $\mathrm{i}$ domini individuati: le risorse psicologiche dei genitori, le caratteristiche del bambino e gli elementi ambientali che possono fungere da fattore di stress o di aiuto ${ }^{4}$. Questi domini sono poi attraversati, a loro volta, da altri fattori quali, ad esempio, la storia evolutiva del genitore, le caratteristiche di personalità e il tipo di relazione coniugale. Belsky ha poi evidenziato che le risorse psicologiche del genitore, il suo benessere psicologico individuale, rappresentano il fattore di protezione più importante nei confronti degli stress derivanti dalla relazione adulto-bambino, superiore perfino alle fonti di supporto sociale e alle caratteristiche del bambino.

Nel racconto di AMF, il fatto di essere cresciuta in una famiglia numerosa l'ha profondamente aiutata ad acquisire quei caratteri che comunemente vengono attribuiti al senso di comunità, e precisamente:

l'appartenenza , cioè la percezione di far parte di un gruppo di persone, di un territorio, di un sistema di simboli; l'influenza e quindi la sensazione di poter incidere con le proprie azioni sui processi di funzionamento della comunità; la soddisfazione dei bisogni cognitivi e valoriali; la

construction de l'ethos, Delachaux et Niestlé S.A., Lausanne (Suisse)-Paris 1999, pp. 155-176.

${ }^{4}$ Belsky J., "The Determinants of Parenting: A Process Model”, Child Development, 55, 1984, pp. 83-96. 
connessione emotiva che rappresenta il legame spirituale e il capitale immateriale, è questa la dimensione che lega il soggetto agli altri membri della comunità. Infatti, ella non esita ad affermare che: "io sono sempre stata molto indipendente anche perché in casa con mia madre ho sempre fatto un po' da mammina ai miei fratelli più piccoli ... anche le attività che venivano fatte, la partecipazione anche sociale, nel paese, la festa paesana, il fulcro di tutto era la nostra casa perché era grande, perché mia madre scriveva le poesie o le commedie, faceva la caccia al tesoro per le feste, si provavano le commedie e quindi c'era sempre un ricambio enorme, poi lo stare a tavola due $\mathrm{o}$ tre ore perché comunque chi è andato a scuola, ma poi magari si tardava dal lavoro e si stava lì a chiacchierare nei confronti di quello che hai fatto a scuola, di quello che hai fatto oggi, è sempre stato un ambiente molto importante questo".

L'ambito familiare di AMF è caratterizzato anche dalla presenza di una figura paterna molto rigida: "Mio padre è uno che gli piace essere lui quello magari che decide, quello che organizza, quello che fa, tenere magari le redini di tutti i figli. Io sono sempre quella che non faccio parte di questo, di essere libera, indipendente...". La necessità di AMF, nonostante i continui e ripetuti riferimenti alla sua volontà di essere autonoma ed indipendente anche dal contesto familiare, è quella di fare riferimento alla sua famiglia di origine per mantenere la stima e la coesione del sé.

In effetti, è assai difficile separare nel soggetto il suo essere dal suo avere poiché il legame tra questi due aspetti è talmente forte che ogni presentazione mobilita, in modo spesso indistinto, l'uno e l'altro aspetto del proprio sé tanto che, mettendo in discussione uno di questi aspetti, il rischio che si corre è quello di rimettere in discussione pure l'altro 5 .

A questo proposito, la scuola di Palo Alto ha, com'è noto, sottolineato che la definizione che il soggetto dà di se stesso può essere oggetto di tre atteggiamenti diversi da parte dell'altro ${ }^{6}$ :

- un atteggiamento di conferma che equivale all'accettazione, da parte dell'altro, della definizione data di se stesso. Questa conferma che al soggetto deriva dall'altro è importantissima perché è capace di garantirgli maturità e stabilità psichiche. Per quanto sorprendente possa sembrare, se non esistesse questo potere di confermare un individuo nella sua identità, la comunicazione umana non avrebbe oltrepassato le frontiere, assai limitate e circoscritte, degli scambi che risultano indispensabili per la protezione e per la sopravvivenza degli esseri umani. Infatti, indipendentemente dal puro e semplice scambio di informazioni, l'uomo ha bisogno di comunicare con gli altri per giungere alla coscienza di sé. Le ricerche sulla privazione sensoriale, che mostrano l'incapacità dell'uomo di preservare una stabilità affettiva se costretto, per periodi prolungati, a comunicare solo con se stesso, forniscono una verifica sperimentale a questa ipotesi. Anche se la privazione ha luogo in un arco di tempo limitato, i suoi effetti possono ripercuotersi

\footnotetext{
5 Bisi R. (a cura di), Scena del crimine e profili investigativi. Quale tutela per le vittime?, FrancoAngeli, Milano, 2006.

${ }^{6}$ Watzlawick P., Helmick Beavin J., Jackson D.D., Une logique de la communication, Éditions Du Seuil, Paris, 1972 ; Kaddouri M., «Le soi : entre présentation et représentation », Education permanente, n.162/2005-1, pp. 9-15.
} 
nel futuro con conseguenze più $\mathrm{o}$ meno rilevanti;

- un atteggiamento di rifiuto che significa che l'altro rifiuta di condividere il contenuto della definizione che diamo di noi stessi. In questo caso, il rifiuto, per quanto penoso possa essere, presuppone che si riconosca almeno in parte ciò che si rifiuta;

- un atteggiamento di negazione che non riguarda né la falsità, né la veridicità della definizione di sé, ma nega la realtà della persona stessa come fonte di questa definizione. Se il rifiuto equivale al messaggio: "Tu hai torto", il messaggio della negazione è: "Tu non esisti".

In tal senso, risultano illuminanti le parole di $\mathrm{W}$. James allorquando afferma che nessuna punizione più diabolica potrebbe essere immaginata rispetto a quella di non essere percepito dai membri della società alla quale si appartiene ${ }^{7}$. E' vero, infatti, che l'interazione dell'uomo con l'ambiente è in grado di determinare il suo comportamento e il suo sviluppo e allorquando l'individuo cambia, muta anche l'ambiente che reagisce alle nostre azioni, ponendo dei limiti oppure sollecitandoci con altri stimoli.

AMF nel corso dei colloqui insiste sulla presentazione, quasi idilliaca, della sua famiglia: "noi avevamo una famiglia bellissima, che eravamo sereni e felici, perché eravamo felici, perché se io non ero felice di stare lassù nelle mie montagne, non ci stavo. Ero libera di prendere e di andare da qualsiasi parte, nessuno mi teneva là. Quella casa l'ho disegnata io, l'ho arredata io con

7 James W., Il flusso di coscienza. I principi di psicologia (a cura di L. Demartis), Mondadori, Milano, 1998. la condivisione di mio marito, ma ero io che vi dedicavo tutto, perché mi piaceva, perché lui aveva il lavoro e pensava a quello e mi lasciava carta bianca in questo, perché siamo così, marito e moglie, ognuno si occupa di qualcosa. A me piaceva, non vedo l'ora di tornarci, e io là ci torno, perché quella è la mia casa, non è qua".

Lo sforzo che ella compie per il perseguimento di un'autogratificazione coinvolge e disperde molte energie facendola vivere in stato di allerta permanente e probabilmente facendole sprecare incalcolabili energie per paura, insicurezza, ansia, rabbia, sospettosità.

L'ansia, infatti, "come condizione di allarme permanente, sostiene e rinforza lo stato difensivo della corazza e distorce continuamente i messaggi interni ed esterni",

Attraverso i numerosi colloqui è stato possibile raccogliere innumerevoli indicazioni sui tratti di personalità del soggetto, sulle problematiche più incalzanti, sulle modalità di relazionarsi, sull'immagine che ella ha di se stessa e molti altri elementi che, in ambito criminologico, risultano indispensabili per definire lo stato di mente del soggetto nel momento in cui si eseguono le indagini perché la presenza di disturbi psichici, che possono configurare in alcuni casi una infermità tale da influire sulla capacità di intendere o di volere, può essere l'elemento su cui si fonda un giudizio sul comportamento futuro e quindi sulla eventuale pericolosità sociale ${ }^{9}$.

In definitiva, tali indagini esigono che si giunga a provare quale era lo stato di mente nel momento

\footnotetext{
${ }^{8}$ Craia V., Craia E., Psicopatologia del carattere $e$ disfunzioni sociali, Edizioni Magi, Roma, 2008, p. 220. 9 Bisi R., "La psicodiagnostica in criminologia: orientamenti e prospettive", in Mucciarelli G., Chattat R., Celani G. (a cura di), Teoria e pratica dei test, Padova, Piccin, 2002, pp. 507-527.
} 
in cui un individuo si rese autore di un atto criminoso, talora collocato anche in un passato non recente, che si precisi il suo stato di mente e che, qualora si riconosca questi affetto da una infermità di mente, si emetta un giudizio, cioè una prognosi sul comportamento futuro.

Per giungere a questi giudizi, gli strumenti di indagine sono quelli tipici della psichiatria, della psicologia e, per alcuni aspetti, anche della sociologia dovendo pervenire ad una conoscenza bio-psico-sociale del soggetto. Infatti, i punti fondamentali che devono essere affrontati nell'esplorazione biografica saranno quelli ritenuti utili "per descrivere e comprendere, in senso propriamente ermeneutico, il complesso, intricatissimo insieme di rapporti intercorrenti tra la biografia di un individuo, le caratteristiche di base della sua personalità - ammesso che sia possibile distinguere tra caso e necessità - e il gruppo familiare di origine, gli altri gruppi primari cui ci si può, più o meno stabilmente, legare e infine il quadro globale della più ampia società, con il suo mondo normativo e le sue strutture istituzionali" ${ }^{\prime 10}$.

L'utilità di poter disporre di storie di vita si collega anche all'art. 236 CPP riguardante ' Documenti relativi al giudizio sulla personalità' che precisa: “E' consentita l'acquisizione di certificati del casellario giudiziale, della documentazione esistente presso gli uffici del servizio sociale, degli enti pubblici e presso gli uffici di sorveglianza nonché delle sentenze irrevocabili di qualunque giudice italiano e delle sentenze straniere riconosciute, ai fini del giudizio sulla personalità dell'imputato o della persona offesa dal reato, se il fatto per il quale si procede deve essere valutato in relazione al comportamento o alle qualità morali di questa".

\section{Stabilire una relazione per allentare la tensione.}

I colloqui hanno senz'altro contribuito a delineare AMF nell'insieme dei suoi aspetti di relazioni, di scelte e di storie e di capacità di andare oltre le esperienze maturate conoscendo risorse personali e relazionali.

Il percorso seguito può essere ricondotto ai quattro processi ben evidenziati da Miller e Rollnick $^{11}$ : stabilire una relazione, focalizzare, evocare e pianificare. Lo stabilire una relazione è la base per un rapporto proficuo che porta a focalizzare il motivo per cui la persona è presente al colloquio. Utilizzando le abilità di base del colloquio quali l'ascolto riflessivo, le domande aperte, i riassunti, il sostegno e lo scambio di informazioni, si fanno emergere le motivazioni al cambiamento e si riuniscono le idee e i sentimenti circa le modalità per realizzarlo.

Il processo evocativo si sviluppa, pertanto, attraverso modalità che contribuiscono a far emergere dalla persona il proprio punto di vista sul comportamento e sui fatti che sono stati focalizzati. Pianificare comporta poi l'impegno al cambiamento e alle strategie di azione e la persona deve sentirsi affiancata mentre sceglie le azioni da compiere per mettere in atto il suo processo di cambiamento, di inizio di una nuova fase della vita. In tal senso, dalla documentazione riguardante il trattamento emerge che l'atteggiamento di AMF nei confronti degli

${ }^{10}$ Ferrarotti F., Storia e storie di vita, Laterza, Bari, 1981, pp. 3-4.

${ }^{11}$ Miller W.R., Rollnick S., Il colloquio motivazionale, Erickson, Trento, 2014. 
operatori è stato sempre corretto ed ella ha manifestato il forte desiderio di essere aiutata ad uscire dalla situazione di disperazione con cui si trova a vivere così spesso.

Gli interventi trattamentali effettuati, appunto nel senso del sostegno alla persona, hanno riguardato principalmente il consentire alla stessa di svolgere un'attività lavorativa utile. Ha svolto, a rotazione, l'attività di scrivana per la sezione, è stata poi assegnata con le stesse modalità alla cucina con il ruolo di cuoca/porta vitto e "jolly". In base alle regole che disciplinano le attività lavorative interne, ha lavorato circa una volta ogni due mesi come addetta alla distribuzione del sopravvitto, ruolo che ha svolto con competenza (è diplomata in ragioneria) e diligenza. Ha partecipato al corso di cucito tenuto settimanalmente da volontari. Ha successivamente fatto richiesta di essere inserita in un corso di formazione professionale di "Sartoria e Ricamo" che le ha permesso, durante il lavoro all'esterno del carcere, di prestare la sua opera in un laboratorio sartoriale gestito da una cooperativa sociale.

Anche i rapporti con le altre detenute presenti in sezione sono narrati da AMF ed anche dal personale di polizia penitenziaria come privi di particolari problemi. Certamente all'inizio l'impatto con la realtà del carcere, con le storie personali di altre persone detenute, ha fatto emergere qualche difficoltà, ma in seguito ciò è stato superato anche in virtù di un apprezzabile sforzo da lei prodotto nel farsi accettare.

In tal senso, l'accomodamento cognitivo descritto da Piaget $^{12}$ rappresenta un modello utile per spiegare ciò che avviene quando l'individuo è chiamato a confrontarsi con un evento che esula dal suo abituale contesto di riferimento. Egli è costretto a trasformare i suoi schemi cognitivi o a elaborarne di nuovi. Ciò sottopone l'individuo ad una consistente attività cognitiva di rivalutazione e rivisitazione di modalità e schemi e ciò può comportare un processo molto lungo e impegnativo.

In tal senso, il test di Rorschach di AMF ha messo in evidenza che quando il soggetto si trova solo, in contatto con se stesso, è possibile che si attivino aspetti soprattutto depressivi che favoriscono un irrigidimento dei nessi associativi.

I meccanismi difensivi appaiono evoluti ed orientati alla rimozione non patologica. E' noto che la teoria e l'osservazione psicoanalitica hanno evidenziato che la difesa basata sulla rimozione è parte dello sviluppo e dell'organizzazione normali della personalità adulta. In realtà le personalità adulte che hanno subito fortemente la rimozione hanno solitamente un aspetto infantile, alcune in modo evidente, altre in forma meno percepibile. La loro esperienza emotiva tende "a rimanere piuttosto dispersa e labile; le loro azioni tendono a essere dominate dagli impulsi; la loro angoscia presenta caratteristiche ingenuamente pavide $\mathrm{o}$ fobiche; e i loro rapporti interpersonali, anche se intensi, sono per lo più di tipo narcisistico, con un attaccamento infantile e/o instabili" $" 13$.

Il test di Rorschach di AMF ha, infatti, evidenziato l'emergere, a livello affettivo, di bisogni di contatto, attenzione al corpo, bisogni narcisistici, ma soprattutto tendenze regressive, viste le molte figure caratterizzate come

12 Piaget J., Psicologia dell'intelligenza, GiuntiBarbera, Firenze, 1975.

13 Schafer R., L'interpretazione psicoanalitica del Rorschach, Boringhieri, Torino, 1971, p. 241. 
"cuccioli"14, "elefantini",15, "uccellini",16, "pesciolini",17, "due vermetti",18.

E' fatto ormai assodato che la situazione carceraria possa di per sé divenire uno stato patologico e molti studi hanno evidenziato quella patologia nota come "sindrome da prisonizzazione" che porta il detenuto verso una condizione di dipendenza nei confronti della cura medica. Infatti, all'atto dell'ingresso in un istituto penitenziario viene attivato da parte del detenuto un processo di assimilazione delle norme, dei valori e dei modelli di comportamento propri dell'istituzione dal quale è ben difficile rimanere esclusi, dato che coinvolge interamente diversi livelli dell'esistenza, dal mero bisogno di sopravvivenza fisica alla dimensione propria della facoltà immaginativa.

Come evidenziato da molti medici penitenziari, il tipo di disagio sanitario, infatti, che il carcere, come situazione estrema, crea è la regressione e pertanto il ricorso ad un comportamento inadeguato all'età per evitare responsabilità o richieste da parte degli altri e rendere possibile un atteggiamento di autoindulgenza ${ }^{19}$.

Alcuni medici sottolineano poi la comparsa del fenomeno, definito abitualmente "invalidismo" che può rappresentare, in condizioni di frustrazione, qual è sicuramente il carcere, il ritorno ad un comportamento vantaggioso sperimentato nell'infanzia o in epoche precedenti. Il test di Rorschach di AMF tende ad evidenziare una dotazione intellettiva di base media con un modo di pensare semplicistico, egocentrico, carico

\footnotetext{
${ }^{14}$ A Tav.II.

${ }^{15}$ A tav.IX.

${ }^{16}$ A tav.V.

${ }^{17}$ A tav.X.

18 Ancora a Tav.X.
}

di affettività, si potrebbe forse dire una persona adulta con l'Io di un bambino. Le risposte date alla tavola IX ${ }^{20}$, ad esempio, la tavola che mobilita maggiormente l'inconscio e di più difficile interpretazione perché la sua struttura cromatica e formale non consente un'immediata evidenza percettiva alla quale rifarsi, ha evidenziato un chiaro sentimento esibizionistico e un forte bisogno di centralità. E' questa una tavola che, assieme alle difficoltà interpretative, offre anche l'opportunità al soggetto di esprimere le proprie risorse psichiche.

E' stato possibile effettuare un confronto con il precedente test di Rorschach somministrato nel corso di un altro accertamento tecnico (2003). Il precedente protocollo appariva molto più povero, caratterizzato da una evidente condizione traumatica reattiva nella quale la rimozione aveva fortemente coartato le espressioni di AMF.

Il quadro psicologico che emerge dal test di Rorschach, secondo la valutazione psicodiagnostica effettuata nel $2014^{21}$, risulta più evoluto e stabile per quanto riguarda le espressioni cognitive, adattative ed affettive, segno di maggior contatto con la realtà e sostanziale capacità di adattarvisi.

Aderendo alla convinzione che l'uso di strumenti diversi nell'indagine psicodiagnostica, utilizzata anche in ambito criminologico, può contribuire a delineare i molteplici livelli di funzionamento della psiche fornendo un numero elevato di

${ }^{19}$ Bisi R., Operatori penitenziari a confronto, Clueb, Bologna, 1990.

20 1) Sembra un gruppo musicale, due sassofonisti, il palco, due sassofonisti e le luci; 2) mi dà l'idea anche di due crostacei; 3) e non so perché ma anche due elefantini. 
informazioni utili alla diagnosi che dal confronto fra le prove si arricchisce e trae giovamento, il consulente tecnico di ufficio ha proceduto all'esame della personalità grafica ${ }^{22}$ di AMF. Infatti, quest'ultimo si completa molto bene con il test di Rorschach poiché "la grafologia rivela molto spesso ciò che il test di Rorschach passa sotto silenzio. Per contro, la scrittura non rivela l'attitudine creatrice del genio al contrario del Rorschach che vi perviene qualche volta (prima di tutto dalle $\mathrm{K}$ e dalle orig.); la scrittura non permette di formulare diagnosi psichiatriche, ciò che invece è possibile, se non sempre, almeno più sovente con il Rorschach" ${ }^{\text {,23. }}$.

Dall'analisi della personalità grafica risulta che AMF è molto sensibile al giudizio altrui e, per cercare di risultare bene accetta, assume atteggiamenti coscientemente controllati entro cui si mescolano sia la sensibilità che l'innata tendenza a ricercare la stima e l'approvazione degli altri. Esteriormente può apparire forte, perché è in grado di mascherare le sue debolezze sotto una sostenutezza più apparente che reale, ma interiormente è fragile sia per l'innata sensibilità, sia per le apprensioni improvvise che la portano verso stati di tristezza.

\section{Progetto di vita e modalità di adattamento.}

Le risorse psichiche di AMF e le sue caratteristiche di personalità le hanno comunque permesso di fare riferimento all'abilità $o$ accortezza sociale, intesa come capacità di reagire positivamente alle sollecitazioni con persone

\footnotetext{
21 Valutazione effettuata dal dott. Roberto Cicioni, psicologo e direttore dell'Istituto Rorschach forense, Roma.

${ }^{22}$ Analisi effettuata dal prof. Alberto Bravo, docente a contratto di grafologia peritale e investigativa, Latina.
}

diverse e nelle circostanze più varie, come evidenziato dai rapporti che ha saputo instaurare con le compagne di carcere e le diverse figure professionali operanti nell'istituto penitenziario.

L'esperienza vissuta da AMF, sottoposta ad una grave e prolungata frustrazione, ha senz'altro contribuito allo sviluppo di sentimenti di fallimento personale e di ansia, seguiti da comportamenti volti alla difesa dell'immagine di sé senza dimenticare che la connotazione essenzialmente psicologica dell'identità diviene anche sociale, poiché colloca la persona nell'ambito dei diritti e delle obbligazioni della comunità.

Il percorso che ha portato, nel giugno 2014, AMF ad essere ammessa alla misura alternativa della detenzione domiciliare speciale (ai sensi dell'articolo 47-quinquies dell'ordinamento penitenziario) rappresenta un esempio dell'importanza rivolta alle relazioni, ai processi ed anche alle modalità di costruzione degli interventi, aperti ad una circolarità comunicativa che diviene occasione per nuove combinazioni creative e produttive di senso in cui la valenza interattiva e quindi dinamica lascia aperti ampi spazi alla relazione fra il soggetto e il suo contesto.

In questa prospettiva, la storia di vita di AMF è stata letta ed interpretata nelle relazioni di consulenza tecnica di ufficio medico-legale e psichiatrica come un intreccio di relazioni sociali poiché è ovvio che una matura ed efficiente integrazione della personalità non si raggiunge solo alimentando potenzialità innate, bensì richiede un'idonea direzione entro un adeguato ambiente_interpersonale.

23 Bohm E., Manuale di psicodiagnostica di 
Infatti, riconoscere all'individuo una sua identità come singola persona significa attualizzare concretamente il principio ed il valore di cui è portatore.

In tale accezione il concetto di identità trova la sua piena realizzazione sul piano delle pratiche di vita attraverso le quali l'uomo si mette in relazione con il mondo mediante il suo conoscere e il suo agire.

Il sé quindi non risulta soltanto dalla coscienza del soggetto pensante, ma, riprendendo William James, è formato da un insieme di elementi che, attraverso il fluire costante del pensiero, vengono sentiti dalla persona come suoi: il corpo innanzitutto, le persone più care, i ruoli sociali e così via.

"Il confine tra ciò che una persona chiama me stesso e ciò che chiama semplicemente mio è difficile da tracciare. Noi sentiamo e agiamo in rapporto a certe cose che sono nostre in modo molto simile a come sentiamo e agiamo in rapporto a noi stessi" ${ }^{24}$.

In ogni caso e "nel suo senso più ampio il sé di un uomo è la somma totale di tutto quello che egli può chiamare suo: non soltanto il suo corpo e le sue facoltà psichiche, ma anche i suoi indumenti e la sua casa, sua moglie e i suoi figli, i suoi antenati e i suoi amici, la sua reputazione e le sue opere, le sue terre e i suoi cavalli, lo yacht e il conto in banca. Tutte queste cose gli danno le stesse emozioni. Se crescono e prosperano, si sente trionfante; se deperiscono e diminuiscono, si sente abbattuto - non necessariamente nella stessa

Rorschach, Giunti-Barbera, Firenze, 1969, pp. 22-23.

24 James W., Il flusso di coscienza. I principi di psicologia (a cura di L. Demartis), Mondadori, Milano, 1998, pp. 115-116. misura per ogni cosa, ma pressappoco allo stesso modo per tutte",25.

Se il primo livello del sé è quello che James definisce sé materiale, in cui il proprio essere e alcune particolari realtà sono identificati, in stretta correlazione con questo vi è il sé sociale, ossia il sentimento della considerazione che gli altri hanno di noi: "Il sé sociale di un uomo è il riconoscimento che egli riceve da chi gli sta intorno" 26 .

Infatti, ove fosse materialmente possibile, non si potrebbe intravedere una soluzione più malvagia di quella per cui un uomo, libero di muoversi nella società, passasse completamente inosservato di fronte a tutti i suoi componenti.

Affermare che lo sguardo dell'altro, l'immagine di noi che vediamo rispecchiata negli individui che ci stanno intorno, partecipa alla formazione del nostro modo di essere, significa pensare l'essere umano come un insieme di relazioni con l'ambiente: in particolare con le cose (come emerge dall'analisi del sé materiale) e con ciò che sentiamo più simile a noi, appunto le persone.

Per questa ragione James sosteneva che "un uomo ha tanti sé sociali quante sono le persone che lo riconoscono [...]. Scalfire una sola di queste immagini equivale a scalfire lui stesso"27. Da ciò deriva la divisione della persona in numerosi sé e si può trattare di una divisione discordante, come allorquando una persona teme che le sue conoscenze di un certo ambiente lo vedano come è altrove oppure, al contrario, può essere una divisione del lavoro perfettamente riuscita, come quando una persona, affettuosa nei confronti dei

\footnotetext{
${ }^{25}$ Ibidem, p. 116.

${ }^{26}$ Ibidem, p. 120.

${ }^{27}$ Ibidem, p. 121.
} 
figli, diviene inflessibile nei confronti di coloro che devono sottostare ai suoi comandi.

In tale prospettiva, il senso della nostra individualità e unicità personale può essere allora considerato il prodotto dell'equilibrio dinamico tra la "tendenza verso l'esterno", volta a cogliere il nostro essere parte di un tutto, e la "tendenza verso l'interno", volta a percepire la totalità del nostro essere una parte. Pertanto, l'interazione diretta e simbolica con l'esperienza degli altri diviene il processo basilare capace di trasformare il divenire soggettivo in un'operazione incessante di assimilazione di esperienza personale.

La percezione della nostra identità personale, corrispondente poi al senso stesso della realtà, trova quindi negli altri la possibilità di esistere e, al contempo, scopre nel processo di differenziazione dagli altri il presupposto, parimenti necessario, per poter giungere ad avere un'esperienza di sé.

Il rapporto dell' Io con se stesso è sempre anche un rapporto con le cose e con gli altri. Di questo rapporto noi portiamo i segni, le difficoltà e le ferite poiché l'unità dell' Io non è mai assoluta e il rapporto con gli altri è spesso problematico.

La prospettiva dalla quale osservare rappresenta un ulteriore fattore critico nel senso che ciò significa presumere che la competenza, l'efficacia e la correttezza di un intervento risultano valutabili da testimoni che non sono imparziali, ma che hanno interessi ed aspettative precisi.

In altri termini, nella valutazione delle azioni di coloro che occupano una particolare posizione specialistica o professionale che li legittima ad intervenire sulla realtà sociale, è importante accordare la massima attenzione alla possibilità di soddisfare i bisogni e le aspettative presenti negli ambiti in cui agiscono. E' questa possibilità, infatti, che viene sottoposta ad analisi critica da parte di soggetti dotati di un'autonoma capacità d'interpretazione.

Ci si trova di fronte pertanto ad una modificazione di rapporti sociali in cui la presenza di rischi che non possono essere eliminati è destinata a mettere in discussione il rapporto "libertà-sicurezza" e a rendere sempre più difficile l'arduo compito di arrestare la crescita di rischi senza limitare le nostre libertà più preziose. E' evidente che queste considerazioni non possono fornire soluzioni immediate, ma credo debbano essere tenute presenti allorquando ci si occupa del posto che può essere riservato alla persona, anche quella condannata per delitti efferati, nella società attuale: l'impegno dovrà essere quello, infatti, di valorizzare ed affermare la dignità dei sentimenti e delle relazioni affettive, utili per ridare senso alla propria appartenenza sociale, nel tentativo di conciliare le ragioni del cuore e le passioni della ragione.

Attività, azioni concrete, quindi un fare che, per risultare soddisfacente, non può mai essere disgiunto dallo stare. Lo stare col pensiero per poi fare è una possibilità che abbiamo per trasformare le relazioni e che ci riporta ai fondamenti della nostra socialità e del vivere in comunità, che ha caratterizzato le relazioni di consulenza tecnica di ufficio medico-legale e psichiatrica nel "caso Cogne" nella consapevolezza che sarebbe stato senz'altro più facile procedere sul vecchio percorso anziché riattivare e prestare ascolto all'autenticità sollecitata dall'esperienza traumatizzante e dolorosa che ha sconvolto le aspettative personali. 
I percorsi psicologici possono essere alternativamente lenti o rapidi e ciò è strettamente correlato alle persone, ai momenti, alle fasi della vita. E' per questa ragione che anche la più grande delle catastrofi, per non spingerci all' autoeliminazione, deve fornirci l'occasione per riflettere sulla nostra vulnerabilità confidando sull'aiuto dell'ambiente esterno che dovrà fornire gli elementi necessari alla realizzazione di un nuovo inizio.

\section{Bibliografia di riferimento.}

- Arcuri L., «Rappresentazione di sé ed elaborazione dell'informazione sociale », in Caprara G.V. (a cura di), Personalità $e$ rappresentazione sociale, La Nuova Italia Scientifica, Roma, 1988.

- Belsky J., "The Determinants of Parenting: A Process Model", Child Development, 55, 1984.

- Bisi R., Operatori penitenziari a confronto, Clueb, Bologna, 1990.

- Bisi R., "La psicodiagnostica in criminologia: orientamenti e prospettive", in Mucciarelli G., Chattat R., Celani G. (a cura di), Teoria $e$ pratica dei test, Padova, Piccin, 2002.

- Bisi R. (a cura di), Scena del crimine e profili investigativi. Quale tutela per le vittime?, FrancoAngeli, Milano, 2006.
- Bohm E., Manuale di psicodiagnostica di Rorschach, Giunti-Barbera, Firenze, 1969.

- Craia V., Craia E., Psicopatologia del carattere e disfunzioni sociali, Edizioni Magi, Roma, 2008, p.220.

- Ferrarotti F., Storia e storie di vita, Laterza, Bari, 1981.

- Goffman E., La mise en scène de la vie quotidienne. La présentation de soi, Les Éditions de minuit, Paris, 1973.

- Haddad G., «Ethos préalable et ethos discursif: l'exemple de Romain Rolland », in R. Amossy (sous la direction de), Images de soi dans le discours - La construction de l'ethos, Delachaux et Niestlé S.A., Lausanne (Suisse)-Paris, 1999.

- James W., Il flusso di coscienza. I principi di psicologia (a cura di L. Demartis), Mondadori, Milano, 1998.

- Kaddouri M., «Le soi : entre présentation et représentation », Education permanente, n.162/2005-1, pp. 9-15.

- Miller W.R., Rollnick S., Il colloquio motivazionale, Erickson, Trento, 2014.

- Piaget J., Psicologia dell'intelligenza, GiuntiBarbera, Firenze, 1975.

- Schafer R., L’interpretazione psicoanalitica del Rorschach, Boringhieri, Torino, 1971.

- Watzlawick P., Helmick Beavin J., Jackson D.D., Une logique de la communication, Éditions Du Seuil, Paris, 1972. 